\title{
Manufacturing Method for Customizable Collagen Membrane Scaffolds for Use in Tissue-Engineering Applications
}

\section{María Dolores Montalvo-Parra}

Tecnologico de Monterrey: Instituto Tecnologico y de Estudios Superiores de Monterrey

\section{Wendy Ortega-Lara}

Tecnologico de Monterrey: Instituto Tecnologico y de Estudios Superiores de Monterrey

Cesar E. Calzada-Rodríguez

Tecnologico de Monterrey: Instituto Tecnologico y de Estudios Superiores de Monterrey

\section{Guiomar Farid Torres-Guerrero}

Tecnologico de Monterrey: Instituto Tecnologico y de Estudios Superiores de Monterrey

\section{Betsabé Hernpandez-Sedas}

Tecnologico de Monterrey: Instituto Tecnologico y de Estudios Superiores de Monterrey

Judith Zavala ( $\boldsymbol{\nabla}$ judith.zavala@tec.mx )

Tecnologico de Monterrey: Instituto Tecnologico y de Estudios Superiores de Monterrey

Italia Tatnaí Cárdenas-Rodríguez

Tecnologico de Monterrey: Instituto Tecnologico y de Estudios Superiores de Monterrey

Jorge E. Valdez-García

Tecnologico de Monterrey: Instituto Tecnologico y de Estudios Superiores de Monterrey

\section{Methodology}

Keywords: scaffold, collagen membrane, tissue engineering, synthesis method

Posted Date: June 8th, 2021

DOI: https://doi.org/10.21203/rs.3.rs-575570/v1

License: (1) (1) This work is licensed under a Creative Commons Attribution 4.0 International License. Read Full License 


\section{Abstract}

\section{Purpose}

Collagen scaffolds are used as tissue engineering tool. The manufacturing methods often lack of reproducibility which limits their application to regenerative medicine. We aimed to set a simple and reproducible method for the production of collagen scaffolds for tissue engineering.

\section{Methods}

A matryoshka system was built comprising a sealed desiccator containing a saturated $\mathrm{K}_{2} \mathrm{CO}_{3}$ solution for a $40 \%$ relative humidity $(\mathrm{RH})$ regulation, this was placed inside a $40^{\circ} \mathrm{C}$ shaking incubator. Collagen gels were cast with a 1:1 ratio of Dulbecco's Modified Eagle's medium and $0.5 \mathrm{mg} / \mathrm{mL}$ acid collagen solution. Three gel volumes were selected: $2.2 \mathrm{~mL}(1 \mathrm{x}), 4.4 \mathrm{~mL}(2 \mathrm{x})$, and $6.6 \mathrm{~mL}(3 \mathrm{x})$. Collagen gels were inserted into the system and desiccation was performed over 37 days (6-12 $\mathrm{mL}$ was desiccated per cycle). Characterization of the surface, ultrastructure, transparency, composition, and biocompatibility of the gels was performed using optical, 3D confocal, and scanning electron microscopy; spectrophotometry; Fourier-transform infrared spectroscopy; and X-Ray diffraction.

Results

On day 7 , collagen membranes exhibited superficial fibrils of $1.3 \mu \mathrm{m}(\mathrm{SD}, \pm 0.23 \mu \mathrm{m})$, whereas on day 37 , a highly organized laminar structure was observed within a smooth homogeneous surface. Lamina density and organization and membrane width (3.65 $\mu \mathrm{m}[1 \mathrm{x}], 4.8 \mu \mathrm{m}[2 \mathrm{x}]$, and $7.2 \mu \mathrm{m}[3 \mathrm{x}])$ increased with gel volume. Transmittance ranged from $77 \%$ to $99 \%$ and increased with wavelength at UV-vi. Gels at $1 x$ and $2 x$ exhibited a $99 \%$ transmittance peak at the green wavelength. The population of cells cultured on membranes was increased by threefold within $48 \mathrm{~h}$; moreover, the size of cell populations cultured on $1 \mathrm{x}$ membranes increased by $12 \%$ compared with the control.

\section{Conclusions}

The scaffolds produced by the matrioshka system were biocompatible, non-cytotoxic, and optically transparent. These membranes can be tailored for multiple uses by modifying their thickness with the volume of the gel and its desiccation time.

\section{Background}

The production methods that have been reported for collagen scaffolds include desiccation, freeze drying, use of hydrogels, electrospinning, and printing [1-6]. These physical and mechanical treatments are applied for the production of collagen hydrogels, membranes, and hybrid polymers. Collagen gels provide 3D scaffolding, whereas collagen membranes are suitable for layered regenerative purposes. However, the lack of a reproducible manufacturing method for collagen scaffolds is an obstacle to their 
application to tissue engineering (TE). Here, a simple method was described for bovine type 1 collagen membrane production using specific gelation and desiccation conditions.

Variations in desiccation conditions have been reported in this context. Collagen vitrigel, a membrane resulting from desiccated collagen gel, was originally proposed by Takezawa et al., who used stabilization and desiccation conditions for the production of a transparent material [6]. Subsequently desiccation allowed the obtaining of a membrane with a thickness of $20-50 \mu \mathrm{m}$ and similar transparency characteristics [7]. Further studies of the interaction among time, relative humidity (RH), and temperature, enabled the customization of desiccated collagen membranes by indicating the main factors ruling material thickness, fiber diameter, density, and organization [8]. This enables a range of desirable characteristics to be applied in different TE fields [9-15].

Tailoring collagen scaffolds for organ design and reconstruction via TE represents an important breakthrough that may alleviate the organ and tissue scarcity reported by the World Health Organization [17-19]. In addition to organ-donor shortage, allograft transplantation also has disadvantages regarding patient recovery and outcomes [20]. In some cases, such as nerves, bone, cartilage, skin, and tendons, autografts are a useful option for diminishing immunological compromise. In both cases, neither mechanical resistance nor tissue regeneration is assured. Moreover, the harvesting of allografts and autografts is an expensive process that is constrained by the anatomy and may transmit infection or disease from the donor to the recipient [26-30]. Therefore, the solutions available currently are partial and insufficient [21-26].

Regenerative medicine or TE stemmed from the interest in improving, maintaining, substituting, and restoring biological tissues [30], even though some authors mark its origins on biomaterial development [31]. Inherently, TE implies the use of scaffolds for cell growth to assure signals, biological stimuli, and host minimal (or no) immune reaction. Thus, certain biomaterial requirements, such as biocompatibility, biodegradability, mechanical properties, and scaffold architecture, must be procured [5, 32], all of which are planned for adaptation to the tissue to be repaired or replaced.

Collagen is ideal for tissue creation by TE because it possesses low immunogenicity, produces porous structures, allows permeability, has good biocompatibility, and is biodegradable. As the most abundant protein in the extracellular matrix, collagen is also readily available. In addition, collagen promotes cell adhesion and migration. Nevertheless, this protein shows poor mechanical strength and stability, which limit its application in regenerative medicine. Thus, intermolecular crosslinking has been performed to increase the structural strength of collagen [16]. Consequently, the collagen-scaffold manufacturing technology must be detailed and registered to provide a standard for good manufacturing practices, which will reduce the time gap from the development of prototypes to their clinical applications [33]. Hence, a simple method with specific gelation and desiccation conditions is necessary for bovine type 1 collagen membrane synthesis.

\section{Results}




\section{Matryoshka system}

The temperature inside the system remained constant at $40^{\circ} \mathrm{C}$ (SD of $\pm 0.19^{\circ} \mathrm{C}$ at the stabilization phase and $\mathrm{SD}$ of $\pm 0.64^{\circ} \mathrm{C}$ in the presence of the samples). The $\mathrm{RH}$ at the stabilization phase ranged from $47-$ $42 \%$ (SD, $\pm 1.68 \%$ ), whereas it ranged from $51-33 \%$ (SD $\pm 5.52 \%$ ) during the sample-desiccation period (Fig. 1b, c). A volume of $50 \mathrm{~mL}$ of the saturated salt solution lasted for $\sim 100$ days when the samples were inside the matryoshka system. Shaker function was turned on $48 \mathrm{~h}$ after the gel was placed inside the matryoshka system, to avoid gel tilting.

\section{Desiccation}

The transformation of gels (Fig. 2a) into membranes occurred when the thickness of the collagen decreased to $\sim 1 \mathrm{~mm}$. Gels at $1 \mathrm{x}$ required $10-13$ days to reach the membrane state, whereas $2 \mathrm{x}$ and $3 \mathrm{x}$ required approximately 15 and 20 days, respectively. We also discovered that phenol red must be removed during the gel-to-membrane transformation, to avoid further interference with transparency (Fig. 2a, b). At the end of the 37-day desiccation period, the membranes were completely transparent (Fig. 2c).

\section{Membrane rehydration}

Membrane rehydration for $\sim 20$ min was necessary to achieve a good retrieval from the cast. First, the borders of the membrane were lifted, then the border was scratched gently with a rounded spatula. No support was needed to retrieve the membranes from the casts. A malleable, transparent material was observed in water suspension. The membranes were placed in a water drop on plastic film, the borders were unfolded, and water was removed using a pipette, to straighten the membranes, which were left to dry again at room temperature (Fig. 2c).

\section{Confocal microscopy}

Confocal microscopy of the material obtained was performed at a width of $3.65 \mu \mathrm{m}$ for $1 \mathrm{x}$ collagen membranes, $4.8 \mu \mathrm{m}$ for $2 \mathrm{x}$ membranes, and $7.2 \mu \mathrm{m}$ for $3 x$ membranes (Fig. 3). Our method produced homogeneous surfaces. The deposition of a greater volume of collagen increased membrane width.

\section{Optical microscopy and SEM surface structure}

A homogeneous surface was observed using optical microscopy; however, transparency was enhanced when the membranes were wet (Fig. 4a, b). Moreover, the surface structure changed over the desiccation process: collagen fibers were observed at day 7 but had disappeared from the surface at day 37 (Fig. 4c, $b, e, f)$. The average fiber diameter was $1.3 \mu \mathrm{m}(S D \pm 0.23)$. Surface homogeneity and lack of roughness also increased with increasing collagen concentration (Fig. 4d, e, f).

\section{SEM transversal-view laminar structure}


Membrane width increased with collagen concentration and lamina density (Fig. 5a, b, e, f). Laminar structure was conserved at the three concentrations (Fig. 5b, e, f). Hydration conditions led to lamina fusion (Fig. 5c, d).

\section{In vitro cell adherence, viability, and cytotoxicity}

NIH3T3 mouse fibroblasts showed adherence to $1 x, 2 x$, and $3 x$ membranes. A fluorescence analysis indicated that the $10 \times 10^{3}$ cells that were seeded initially proliferated to $\sim 31.7 \times 10^{3}$ cells in wells with no membranes. The populations of cells cultured on $1 x, 2 x$, and $3 x$ membranes increased to $\sim 35.8 \times 10^{3}$, $29.6 \times 10^{3}$, and $29.5 \times 10^{3}$ cells, respectively. The populations of cells increased by 3-fold within $48 \mathrm{~h}$. One-way ANOVA detected no significant differences between the means of all four samples $(P=0.323, \mathrm{n}$ =3). However, compared with controls, the populations of cells cultured on $1 \mathrm{x}$ membranes increased by $12 \%$. Consistently, we can assume the absence of a cytotoxic effect because the Cell Titer Blue reaction exhibited an inverse correlation with the LDH assay.

\section{Fourier-transformed infrared spectra}

The transmittance peak at wavelength 3281 (Fig. 6, arrow 1) was decreased in 3x collagen membranes, indicating the formation of $\mathrm{O}-\mathrm{H}$ bonds. Moreover, the fingerprint area corroborated the identity of collagen in membranes. Peaks 1660 (Amide I band), 1627, 1635 ( $\beta$-sheet 2ry structures of Amide I), 1637 (triple helix), and 1679 (stretching $\mathrm{C}=\mathrm{O}$ vibrations that are $\mathrm{H}$ bonded) were fused into a peak with least transmittance at 1635 (arrow 2). Peak 1635 indicating $\beta$-sheet secondary structures were correlated with the laminar structure shown in transversal SEM sections. The peak at 1535 (arrow 3) indicated Amide II, whereas the peak at 1240 (arrow 6) was characteristic of collagen. The contribution of peaks at 1446, 1396, and 1202 is seldom (or not at all) reported in the literature (arrows 4, 5, and 7). Peaks 1084 and 1029 (arrows 8 and 9) are related to nucleic acids. Peak 1084 was reported by Movasaghi as corresponding to the phosphodiester bonds of the phosphate/sugar backbone of nucleic acids, whereas peak 1029 was reported as corresponding to collagen and the phosphodiester groups of nucleic acids. Finally, the peaks at 565 and 527 (arrow 10) corresponded to phenyl group torsion. All peaks were intensified as collagen volume increased.

\section{Transmittance analysis}

The membranes produced using our method were 90\% transparent in the visible light spectrum (Fig. 7). The average transmittance for $1 x$ membranes was $92.6 \%$, whereas it was $94 \%$ for $2 x$ and $89.21 \%$ for $3 x$ membranes. Lower transmittance values were recorded at violet wavelengths (ranging from 380 to 450 $\mathrm{nm}$ ); $1 \mathrm{x}$ membranes, $80.4 \%$; $2 \mathrm{x}$ membranes, $82.98 \%$; and $3 \mathrm{x}$ membranes, $77.56 \%$. Moreover, $1 \mathrm{x}$ membranes yielded a transmittance peak of $99.6 \%$ at $560 \mathrm{~nm}$ and $2 x$ membranes had a peak of $99 \%$ at $540 \mathrm{~nm}$, both of which are in the green wavelength spectrum. In contrast, $3 x$ membranes exhibited no peak but yielded higher transmittance values $(94.7 \%)$ at $650 \mathrm{~nm}$. Percent transmittance increased progressively and stabilized at $510 \mathrm{~nm}(1 \mathrm{x})$ ad $590 \mathrm{~nm}(2 \mathrm{x}$ and 3x). Increasing collagen concentration 
decreased transmittance by $\sim 2.5 \%$ per added volume, with $1 \mathrm{x}$ membranes showing the highest percent transmittance. ANOVA indicated the presence of significant differences $(P \leq 0.001)$ for $3 x$ compared with $1 \mathrm{x}$ and $2 \mathrm{x}$ membranes.

\section{X-ray diffraction analysis}

The XRD patterns of pure collagen type $1(1 x, 2 x$, and $3 x$ membranes) are shown in Fig. 8 . All samples exhibited a typical broad hump around $22^{\circ}$, indicating that collagen was in an amorphous phase [34, 35]. A high-intensity peak was observed at $25.9^{\circ}$, which was related with the hydroxyapatite (HA) 002 plane. A shoulder of the HA 320 plane in the hump was shown at $39.8^{\circ}$ compared with datasheet 9-432 from the Joint Committee of Powder Diffraction Standards. All samples showed a similar pattern, regardless of the content of collagen.

\section{Discussion}

Various types of equipment, often specialized and expensive, are required for the production of collagen membrane scaffolds. The main synthesis methods used in this field involve electrostatically charged trays and vacuum systems [36], electrospinning and lyophilization apparatuses [5], and even dialysis equipment [37]. Methods that require a desiccation process include equipment such as humidity chambers and clean air chambers $[6,8]$ with controlled $\mathrm{RH}$; however, no model or further description is provided for these systems [38, 39]. In addition, the use of patented materials has been reported [15].

The matryoshka system is a closed desiccation device that provides a stable and gradual decrease in $\mathrm{RH}$ inside the desiccation bubble regardless of the environmental $\mathrm{RH}$. This ensures system and synthesis stability. Hence, the characteristics of the scaffolds produced using this system are reproducible.

The system described here can be assembled using common laboratory equipment and materials (a desiccator, salts, and a shaking incubator), thus avoiding additional production costs. Our desiccation system exhibited an $\mathrm{RH}$ variation of $5.52 \%$, which was similar to the 5-8\% variation reported for commercial options of higher prices.

Based on desiccation conditions established previously [8], our synthesis process was carried out at $40^{\circ} \mathrm{C}$ and $40 \%$ RH over 37 desiccation days and produced collagen-based membranes that were comparable with previously described materials. Differences in width were addressed first. A width of $20-50 \mu \mathrm{m}$ is obtained after 2 weeks of desiccation using a collagen concentration of $2 \mu \mathrm{l} / \mathrm{mm}^{2}[6,7]$, whereas our membranes were 3-7 $\mu \mathrm{m}$ thick, depending on collagen volume deposition (Figs. 4, 5). The width of the scaffolds was also smaller than that of other materials of different origins and compositions, such as amniotic membranes [7], human purified type I collagen membranes [40], collagen-silk-fibroin [38], crosslinked collagen-gelatin-hyaluronic acid biomimetic films [41], and decellularized and decalcified fish-scale-derived extracellular matrix [42]. A literature review revealed that oriented-flow cast collagen laminates alone attained a thickness of $2-5 \mu \mathrm{m}$ thickness per stacked lamina [43]. 
Our SEM data pertaining to surface and transversal ultrastructure agreed with those reported by other authors, regardless of the use of additional collagen volume in the production of the membranes. Randomly arranged fibrils are observed at the surface of the membranes after short desiccation periods $[7,8]$. Nevertheless, the fibers observed here were larger by an order of magnitude, $1.3 \mu \mathrm{m}(\mathrm{SD}, \pm 0.23)$, compared with the sizes of collagen membranes reported previously $[8,44]$ and resembled the early fibrils reported for collagen gels [45]. This phenomenon is attributed to the different order of the neutralization and blending steps used for gel formation, which triggers the formation of collagen fibrils $[45,46]$. Moreover, the incubation conditions affect the increase in the thickness of fibrils: the lateral fusion of discrete $(\sim 4 \mathrm{~nm})$ subunits leads to an increase in fibril diameter, followed by longitudinal growth [47]. Fibril bundling and total unification (greater fibril density and homogeneity) were observed when drying time was increased and high temperatures were used, leading to the production of the homogeneous and smooth surface observed in $1 x, 2 x$, and $3 x$ membranes. A similar non-fibrillar surface was observed in collagen membranes crosslinked with 1-ethyl-3-(3-dimethyl aminopropyl) carbodiimide (EDC) and Nhydroxysuccinimide (NHS) $[38,48]$ and UV [40]. Heat energy is involved in the process of fibril unification. The transversal laminar arrangement is also conserved across pure collagen-based membranes produced using similar systems, although desiccation time, $\mathrm{RH}$, and temperature vary $[38,39]$.

The highly organized fibril laminae observed transversally were responsible for the high transmittance of our collagen membranes, which was higher than that of the human cornea [40] and those of membranes obtained using comparable conditions $\left(40 \% \mathrm{RH}, 40^{\circ} \mathrm{C}, 1\right.$ week and 5 weeks), as reported by CalderonColon et al. Similar conditions reportedly improved the transmittance of Takezawa's first collagen membrane. However, even at similar collagen concentrations, the transmittance pattern of $1 x$ and $2 x$ membranes was different from those obtained using the different experimental conditions described by Calderon-Colon et al. and from those of all materials mentioned previously, because of the peak detected at the green wavelength (around $550 \mathrm{~nm}$, at which $\mathrm{L}$ and $\mathrm{M}$ cones are stimulated). In contrast, $3 \mathrm{x}$ membranes exhibited a transmittance pattern that was similar to that of other materials, with no peak observed at $550 \mathrm{~nm}$. Transmittance patterns similar to that observed for our $3 \mathrm{x}$ membranes have been reported for other collagen membranes [38, 40], combinations of silk-fibroin [38], chitosan [15], gelatin and hyaluronic acid [41], and fish-scale collagen scaffold [42]. The increase in collagen deposition (i.e., membrane width) sets a light transmittance threshold [43] and pattern. Therefore, the tailoring of the ultrastructure of these membranes by changing desiccation conditions and collagen amount allows the convenient adaptation of their optical transmittance [8].

Furthermore, because of our desiccation conditions and based on previous knowledge [8], we should expect a change in the bulk refractive index, because it depends on the composition and water content of the material. We analyzed the water content of our membranes using FTIR (Fig. 1, peak 3281, arrow 1); we found that it increased with the addition of collagen volume, which was most evident in the case of $3 x$ membranes. It is likely that that additional water molecules are trapped because of the increase in the collagen matrix, which cannot be compensated, even by prolonged desiccation period. Moreover, in contrast with the report of Calderon-Colon et al., we did modify the composition of membranes by adding collagen volume and maintained the desiccation parameters. Therefore, we preserved the structural 
motifs by increasing the number/density of the fibrils (the peak indicated the presence of $\beta$-sheets at FTIR $1635 \mathrm{~nm}$ ), which increased light scattering, thus explaining the lower transmittance observed for $3 \mathrm{x}$ membranes.

Desiccation time could set a volume decay tendency. We understand that the specimens reported by Calderon-Colon et al. had a variable thickness; therefore, they had to be normalized to $50 \mu \mathrm{m}$ to enable comparisons (but the same amount of collagen was used for all conditions). Thus, we confirmed that membrane thickness is a function of the different desiccation conditions [8]. However, our $1 \mathrm{x}$ membranes at 1 week of desiccation were not functional: they were too weak even to resist hydration for separation from the cast. Similar observations were reported previously by other authors [40]. Therefore, the collagen membrane manufacturing process should be standardized across the various types of equipment.

Collagen membrane scaffolds have been widely reported as being biocompatible in vivo and in vitro [7, $10,15,36,38,40,49-51]$. In vitro, our membranes were not cytotoxic and allowed cell adhesion and growth despite of their smooth surface. Cell interactions with collagen might favor and compensate for adhesion and growth despite the presence of a smooth surface $[52,53]$. Of note, we used NIH3T3 fibroblasts from mouse embryos that had lost contact inhibition. Thus, the assessment of proliferation beyond full confluence was not useful for obtaining a clear idea of specific cell-type behavior and, hence, of tissue regeneration. Nevertheless, the experimental design for such purposes using membranes produced with the desiccation system described here should take into account cell size (smaller cells have smaller pores and lower membrane porosity) and stratification behavior caused by the increase in lamina density and width that accompanied the addition of collagen volume in our scaffolds $[4,32,54$, 55]. For example, if a 3D tissue model is intended, the use of a combination of $1 x, 2 x$, and $3 x$ membranes as different basal lamina could be an option for the adaptation of factors such as cell nutrients and oxygen supply.

Further study of various aspects of our experiments may help solve several common biosafety and quality issues. FTIR peaks corresponding to the presence of the sugar backbone of nucleic acids [56] (Fig. 6, arrows 8 and 9) could be a useful indication of the presence/absence of genomic material through a non-destructive test. However, similar peaks have been reported as corresponding to an ester band $(1100 \mathrm{~cm}-1)$ and were indicative of crosslinking [3]. Moreover, the complete FTIR spectrum is not presented often in similar reports of structural characterization (and quantification if absorbance values are provided) and comparisons among samples from different manufacturing rounds. A similar situation exists for the results of 3D confocal microscopy. Finally, findings from the XRD analyses of amorphous collagen and HA provide data pertaining to the origin of the collagen and desirable properties regarding biological responses, such as enhancement of mineralization, long-term degradation, and tissue integration $[57,58]$.

\section{Conclusion}


The specification of type of equipment, gel formation, and desiccation conditions is vital for the reliable, replicable, and uniform production of transparent collagen-based membrane-like scaffolds. This information is useful for customization purposes when addressing the various necessities of TE and regenerative medicine, such as transparency and stacking (for 3D culture). We intended to report detailed information for the use of these applications by other researchers. Moreover, information about the manufactured collagen material may clarify the effects of the width and other properties of membranelike collagen-based materials. We synthesized a biocompatible, transparent, sandwich-like and laminar collagen-based membrane ultrastructure with a smooth surface and a thickness of 3-7 $\mu \mathrm{m}$. Future work should address the effects of these characteristics in different cell lines, primary cultures, and tissues.

\section{Methods}

\section{Matryoshka system}

The matryoshka system consisted of an incubator with a steady temperature $(T)$ bearing a desiccator with a saturated $\mathrm{K} 2 \mathrm{CO} 3$ solution, to control RH. First, $50 \mathrm{~mL}$ of a $\mathrm{K} 2 \mathrm{CO} 3$ solution were prepared using $1.15 \mathrm{~g}$ of K2CO3 (Sigma-Aldrich, P5833, St. Louis, Missouri, USA) per milliliter of bidistilled water. The salt solution was placed in a $200 \mathrm{~mL}$ beaker and fixed onto a $330 \times 246 \times 262 \mathrm{~mm}$ desiccator with a clear plastic dome (Thermo Scientific Nalgene, 5310-0250, Cleveland, Ohio, USA). A closed system was created by placing the sealed desiccator inside a shaking-plate incubator (I5211DS; Labnet International, Edison, New Jersey, USA) (Figure 1 a). The temperature and shaking speed of the incubator were set to $40^{\circ} \mathrm{C}$ and 30 rpm, respectively. T and RH were measured with a Monitoring Traceable Hygrometer (4040CC; Traceable ${ }^{\circledR}$ Products, Webster, Texas, USA) placed inside the desiccator.

\section{System-stabilization phase}

$\mathrm{T}$ and $\mathrm{RH}$ were registered daily until a temperature of $40^{\circ} \mathrm{C}$ and an $\mathrm{RH}$ of $40 \%$ were recorded consistently for $\sim 7$ days. During this period, no sample was placed inside the system.

\section{Collagen-based membrane production}

\section{Collagen gel preparation}

Based on methods described previously [6-8], advanced Dulbecco's Modified Eagle's medium (DMEM, 1x) (12491-015; Gibco-Thermo Fisher Scientific, Grand Island, New York, USA) was prepared with 1\% penicillin-streptomicyn (15140-122; Gibco) and 8\% qualified fetal bovine serum (26140-179; Gibco) and kept on ice. Subsequently, 22 mM HEPES (15630-106; Gibco) was added and mixed with a cold type-I collagen solution $(5 \mathrm{mg} / \mathrm{mL}$ ) at a 1:1 ratio (A10644-01, Gibco) until a uniform yellow mixture was obtained by gentle pipette resuspension. The pipettes were also kept on ice, to avoid early gelification. Twelve-well plates were used as casts for $2.2 \mathrm{~mL}$ of the collagen mix $(2.8 \mathrm{dl} / \mathrm{mm} 2$ of collagen; $1 \mathrm{x}$ volume). Gel casts were also prepared at volumes of $2 x$ and $3 x$. The plates containing the collagen mix 
were placed in an MCO-18AIC incubator (Sanyo, Osaka, Japan) and kept at $37^{\circ} \mathrm{C}, 5 \% \mathrm{CO} 2$ for $2 \mathrm{~h}$, until gelification. No support ring was included.

\section{Desiccation}

Plates containing collagen gels were placed inside the matryoshka system. The temperature and shaking speed of the incubator were set at $40^{\circ} \mathrm{C}$ and $30 \mathrm{rpm}$, respectively. The system remained closed for daily $\mathrm{T}$ and $\mathrm{RH}$ registration. Around day $10-13$, the volume of the collagen gels decreased and membranes were formed. The matryoshka system was then opened and membranes were rinsed with bidistilled water until elimination of the phenol red in the medium. The samples were placed back inside the system, to complete a period of 37 days of incubation.

\section{Membrane rehydration}

Plates were removed from the matryoshka system. Bidistilled water was poured into the wells of the plates containing the collagen membranes, left for $\sim 20 \mathrm{~min}$, then removed. The borders of each membrane were lifted using water pressure. Rounded-tip forceps were used to gently pull the remainder of the membranes away from the bottom of the well.

\section{Collagen-based membrane characterization}

\section{Optical microscopy}

The surface of collagen membranes was observed with an Axiovert 40 CFL contrast microscope (Carl Zeiss Microscopy, Jena, Germany) and photographed. Membranes were humidified for 20 min and the water excess was then removed.

\section{Scanning electron microscopy (SEM)}

Samples were coated with gold using a Quorum QR150 ES sputtering system (Quorum Technologies, Laughton, United Kingdom). The surface and transversal ultrastructure of 37-day samples was observed and measured with an EVO MA Scanning Electron Microscope (Carl Zeiss Microscopy). Moreover, the fibers of samples that were desiccated for 7 days (4 samples, 34 fibers per sample) were photographed and measured using NIH ImageJ. Briefly, image calibration to microns was performed, followed by selection of linear regions of interest and accumulation using the Analysis>>Measure route.

\section{D confocal microscopy}

Surface regularity and sample thickness were assessed in triplicate using an Axio-CSM 700 50x objective (Carl Zeiss Microscopy). Samples were hydrated for 20 min prior to data collection.

In vitro cell adherence, viability, and cytotoxicity 
Collagen membranes were cut into circles with a diameter of $5 \mathrm{~mm}$, to fit a 96-well plate. Membranes were sterilized with Microdacyn (Oculus Innovative Sciences, CA, USA) and rinsed with sterile water. Water was removed and membranes were left to dry and adhere to the bottom of the wells. NIH3T3 cells were seeded $(\sim 10,000)$ in $100 \mu$ l of DMEM F12 (12491-015; Gibco) and incubated overnight at $37^{\circ} \mathrm{C}, 5 \%$ CO2.

A cytotoxicity and viability test was performed using Cell Titer Blue (Promega, Madison, WI, USA) according to the manufacturer's instructions. Briefly, $20 \mu$ of Cell Titer Blue were added to each experimental, assay control, and sample control well, followed by gentle resuspension and incubation at $37^{\circ}, 5 \% \mathrm{CO} 2$ for $2 \mathrm{~h}$. The same conditions were used to perform a 2-50 x 103 cell fluorescence ladder comparison. The Cell Titer Blue fluorescent signal is produced by resazurin reduction, a viability indicator, which exhibits an inverse correlation with the release of lactate dehydrogenase, a cytotoxicity indicator. Fluorescence was recorded on a Synergy HT spectrophotometer (BioTek, Winooski, VT, USA). Triplicates were tested for each membrane concentration in the experimental condition (membrane + cells), the sample control condition (cells alone), the negative control condition (membrane alone), and the assay control condition (medium + Cell Titer Blue).

\section{Fourier-transformed infrared spectra (FTIR)}

Functional groups were identified on each membrane sample using an infrared spectrophotometer coupled with a Fourier transform Spectrum 400 apparatus (Perkin Elmer, Waltham, MA, USA) and recorded in the wavenumber range of $4000-400 \mathrm{~cm}-1$ at room conditions.

\section{Transmittance analysis}

Absorbance was acquired throughout UV-VIS spectra $(380-700 \mathrm{~nm})$ using a Synergy HT spectrophotometer (BioTek, Winooski, VT, USA) and transformed to transmittance using the BeerLambert law equation. Experiments were performed in triplicate for all collagen volumes.

X-Ray diffraction analysis (XRD)

We used XRD to assess the crystallinity of the collagen membranes. XRD was recorded in the $2 \theta$ range, between $10^{\circ}$ and $85^{\circ}$, with a step size of 0.026 using a PANalytical Empyrean diffractometer (PANalytical, Almelo, Netherlands) and CuKa radiation $(\lambda=1.5406 \AA)$. The voltage applied was $45 \mathrm{kV}$ and the current was $40 \mathrm{~mA}$. For measurements, whole samples were fixed to the corresponding support.

\section{Statistical analysis}

Statistical comparison of the experimental groups was carried out using paired Student's t-tests or analysis of variance (ANOVA). Significance was set at $P<0.05$. Microsoft Excel (2013; Redmond, WA, USA) and Systat Sigma Plot (V. 11; San Jose, CA, USA) were used for data processing, statistical analysis, and graph generation.

\section{Declarations}




\section{Ethics approval and consent to participate}

Not applicable

\section{Consent for publication}

Not applicable

\section{Availability of data and materials}

Not applicable

\section{Competing interests}

The authors declare that they have no competing interests

\section{Funding}

This research was funded by CONACyT Grant PN6558.

\section{Authors' contributions}

MDMP conducted and designed the experiments. JZ designed and supervised the experiments and get the fund for the research. WOL designed and supervised the characterization of the collagen membrane. CECR, GFTG, BHS, and ITCR contributed to the membrane preparation, image analysis, and characterization experiments. JEVG contributed to the research funding and supervised the manuscript.

\section{References}

[1] Lee JE, Park JC, Hwang YS, Kim JK, Kim JG and Sub H. Characterization of UV-irradiated dense/porous collagen membranes: morphology, enzymatic degradation, and mechanical properties. Yonsei Med. J. 2001;42:172-9.

[2] Nakada A, Shigeno K, Sato T, Kobayashi T, Wakatsuki M, Uji M, and Nakamura T. Manufacture of a weakly denatured collagen fiber scaffold with excellent biocompatibility and space maintenance ability Biomed. Mater. 2013;8:45010.

[3] Park SN, Park JC, Kim HO, Song MJ and Suh H. Characterization of porous collagen/hyaluronic acid scaffold modified by 1-ethyl-3-(3-dimethylaminopropyl) carbodiimide cross-linking. Biomaterials. 2002;23:1205-12.

[4] Anguiano M, Castilla C, Maška M, Ederra C, Peláez R, Morales X et al. Characterization of threedimensional cancer cell migration in mixed collagen-Matrigel scaffolds using microfluidics and image analysis. PLoS One. 2017;12:e0171417. 
[5] Eltom A, Zhong G and Muhammad A. Scaffold Techniques and Designs in Tissue Engineering Functions and Purposes: A Review. Adv. Mater. Sci. Eng. 2019;2019: Article ID 3429527.

[6] Takezawa T, Ozaki K, Nitani A, Takabayashi C and Shimo-Oka T. Collagen vitrigel: a novel scaffold that can facilitate a three-dimensional culture for reconstructing organoids. Cell Transplant. 2004;13:463-73.

[7] Mclntosh Ambrose W, Salahuddin A, So S, Ng S, Márquez SP, Takezawa T, et al. Collagen vitrigel membranes for the in vitro reconstruction of separate corneal epithelial, stromal, and endothelial cell layers J. Biomed. Mater. Res. - Part B Appl. Biomater. 2009;90:818-31.

[8] Calderón-Colón X, Xia Z, Breidenich JL, Mulreany DG, Guo Q, Uy OM, et al. Structure and properties of collagen vitrigel membranes for ocular repair and regeneration applications. Biomaterials 2012;33:828695.

[9] Lu Q, Al-Sheikh O, Elisseeff JH and Grant MP. Biomaterials and Tissue Engineering Strategies for Conjunctival Reconstruction and Dry Eye Treatment. Middle East Afr. J. Ophthalmol. 2015;22:428-34.

[10] Chae JJ, Ambrose WM, Espinoza FA, Mulreany DG, Ng S, Takezawa T, et al. Regeneration of corneal epithelium utilizing a collagen vitrigel membrane in rabbit models for corneal stromal wound and limbal stem cell deficiency. Acta Ophthalmol. 2015;93:e57-66.

[11] Takezawa T, Nitani A, Shimo-Oka T and Takayama Y. A protein-permeable scaffold of a collagen vitrigel membrane useful for reconstructing crosstalk models between two different cell types. Cells Tissues Organs. 2007;185:237-41.

[12] Nakai S, Shibata I, Shitamichi T, Yamaguchi H, Takagi N, Inoue T, et al. Collagen vitrigel promotes hepatocytic differentiation of induced pluripotent stem cells into functional hepatocyte-like cells. Biol. Open. 2019;8:bio042192.

[13] Oshikata-Miyazaki A and Takezawa T. Development of an oxygenation culture method for activating the liver-specific functions of HepG2 cells utilizing a collagen vitrigel membrane chamber. Cytotechnology. 2016;68:1801-11.

[14] Yamaguchi H, Kojima H and Takezawa T. Vitrigel-Eye Irritancy Test Method Using HCE-T Cells Toxicol. Sci. 2013;135:347-55.

[15] Shen Y, Redmond SL, Teh BM, Yan S, Wang Y, Atlas MD, et al. Tympanic membrane repair using silk fibroin and acellular collagen scaffolds. Laryngoscope. 2013;123:1976-82.

[16] Dong C and Lv Y. Application of collagen scaffold in tissue engineering: Recent advances and new perspectives. Polymers (Basel). 2016;8:42.

[17] O'Brien J, Hayder H and Peng C. Automated Quantification and Analysis of Cell Counting Procedures Using ImageJ Plugins. J Vis Exp. 2016; 117:e54719. 
[18] Girlanda R. Deceased organ donation for transplantation: Challenges and opportunities. World J. Transplant. 2016;6:451-9.

[19] Who.int. 2021. WHO Task Force on Donation and Transplantation of Human Organs and Tissues. [online] available from: https://www.who.int/transplantation/donation/taskforce-transplantation/en/

[20] Justiz Vaillant AA, Waheed A and Mohseni M. Chronic Transplantation Rejection. In: StatPearls Publishing; 2021 Jan-. Available from: https://www.ncbi.nlm.nih.gov/books/NBK535435/

[21] Naik A and Acharya P. Chronic anterior cruciate ligament insufficiency results in inferior quality hamstring autografts. Eur. J. Orthop. Surg. Traumatol. 2019;29:1481-1484.

[22] Vistarini N, Gebhard C, Desjardins G and El-Hamamsy I. Successful Repair of a Bicuspid Pulmonary Autograft Valve Causing Early Insufficiency After a Ross Procedure. Ann. Thorac. Surg. 2016;101:e99101.

[23] Carrel T and Kadner A. Long-Term Clinical and Imaging Follow-Up After Reinforced Pulmonary Autograft Ross Procedure. Semin Thorac Cardiovasc Surg Pediatr Card Surg Annu. 2016;19:59-62.

[24] Pareek A, Reardon PJ, Maak TG, Levy BA, Stuart MJ and Krych AJ. Long-term Outcomes After Osteochondral Autograft Transfer: A Systematic Review at Mean Follow-up of 10.2 Years Arthrosc. J. Arthrosc. Relat. Surg. 2016;32:1174-84.

[25] Safa B and Buncke G. Autograft Substitutes. Hand Clin. 2016;32:127-40.

[26] Vig K, Chaudhari A, Tripathi S, Dixit S, Sahu R, Pillai S, et al. Advances in Skin Regeneration Using Tissue Engineering. Int J Mol Sci. 2017;18:789.

[27] Andes DR, Safdar N, Baddley JW, Alexander B, Brumble L, Freifeld A, et al. The epidemiology and outcomes of invasive Candida infections among organ transplant recipients in the United States: results of the Transplant-Associated Infection Surveillance Network (TRANSNET) Transpl. Infect. Dis. 2016;18:921-31.

[28] Levitsky J, Formica RN, Bloom RD, Charlton M, Curry M, Friedewald J, et al. The American Society of Transplantation Consensus Conference on the Use of Hepatitis C Viremic Donors in Solid Organ Transplantation. Am J Transplant. 2017;17:2790-802.

[29] Fishman JA. Infection in Organ Transplantation. Am. J. Transplant. 2017;17:856-79.

[30] O’Brien FJ. Biomaterials \& scaffolds for tissue engineering. Mater. Today. 2011;14:88-95.

[31] Tissue Engineering and Regenerative Medicine [Internet]. Nibib.nih.gov. [cited 26 May 2021]. Available from: https://www.nibib.nih.gov/science-education/science-topics/tissue-engineering-andregenerative-medicine 
[32] Bružauskaitè I, Bironaitė D, Bagdonas E and Bernotienė E. Scaffolds and cells for tissue regeneration: different scaffold pore sizes-different cell effects. Cytotechnology. 2016;68:355-69.

[33] Hollister SJ. Scaffold engineering: a bridge to where? Biofabrication. 2009;1:012001.

[34] Johnson JR, Meng L, Wnek GE and Schiraldi DA. Electrophoretic calcium phosphate mineralization of collagen hydrogels. Green Mater. 2015;3:71-9.

[35] León-Mancilla BH, Araiza-Téllez MA, Flores-Flores JO and Piña-Barba MC. Physico-chemical characterization of collagen scaffolds for tissue engineering J. Appl. Res. Technol. 2016;14:77-85.

[36] Gigante A, Busilacchi A, Lonzi B, Cecconi S, Manzotti S, Renghini C, et al. Purified collagen I oriented membrane for tendon repair: An ex vivo morphological study J. Orthop. Res. 2013;31:738-45.

[37] Knight DP, Nash L, Hu XW, Haffegee $\mathrm{J}$ and Ho M-W. In vitro formation by reverse dialysis of collagen gels containing highly oriented arrays of fibrils J. Biomed. Mater. Res. 1998;41:185-91.

[38] Long K, Liu Y, Li W, Wang L, Liu S, Wang Y, et al. Improving the mechanical properties of collagenbased membranes using silk fibroin for corneal tissue engineering J. Biomed. Mater. Res. Part A 2015;103:1159-68.

[39] Ambrose WMI, Salahuddin A, So S, Ng S, Márquez SP, Takezawa T, et al. Collagen vitrigel membranes for the in vitro reconstruction of separate corneal epithelial, stromal, and endothelial cell layers J. Biomed. Mater. Res. - Part B Appl. Biomater. 2009;90B:818-31.

[40] Vázquez N, Chacón M, Rodríguez-Barrientos CA, Merayo-Lloves J, Naveiras M, Baamonde B, et al. Human bone derived collagen for the development of an artificial corneal endothelial graft: in Vivo results in a rabbit model. PLoS One. 2016;11:e0167578.

[41] Liu Y, Ren L and Wang Y. Crosslinked collagen-gelatin-hyaluronic acid biomimetic film for cornea tissue engineering applications. Mater Sci Eng C. 2013;33:196-201.

[42] van Essen TH, Lin CC, Hussain AK, Maas S, Lai HJ, Linnartz H, et al. A Fish Scale-Derived Collagen Matrix as Artificial Cornea in Rats: Properties and Potential. Investig Opthalmol Vis Sci. 2013;54:3224.

[43] Tanaka Y, Baba K, Duncan TJ, Kubota A, Asahi T, Quantock AJ, et al. Transparent, tough collagen laminates prepared by oriented flow casting, multi-cyclic vitrification and chemical cross-linking. Biomaterials. 2011;32:3358-66.

[44] Lu Q, Wang X, Lu S, Li M, Kaplan DL and Zhu H. Nanofibrous architecture of silk fibroin scaffolds prepared with a mild self-assembly process. Biomaterials. 2011;32:1059-67.

[45] Kadler KE, Holmes DF, Trotter JA and Chapman JA. Collagen fibril formation. Biochem. J. 1996;316( Pt 1):1-11. 
[46] Holmes DF, Capaldi MJ and Chapman JA. Reconstitution of collagen fibrils in vitro; the assembly process depends on the initiating procedure. Int J Biol Macromol. 1986;8:161-6.

[47] Christiansen DL, Huang EK and Silver FH. Assembly of type I collagen: fusion of fibril subunits and the influence of fibril diameter on mechanical properties. Matrix Biol. 2000;19:409-20.

[48] Nair M, Calahorra Y, Kar-Narayan S, Best SM and Cameron RE. Self-assembly of collagen bundles and enhanced piezoelectricity induced by chemical crosslinking. Nanoscale. 2019;11:15120-30.

[49] Valdez-García JE, Mendoza G, Zavala J, Zavala A, Brito G, Cortés-Ramírez JA et al. In vivo Biocompatibility of Chitosan and Collagen-Vitrigel Membranes for Corneal Scaffolding: a Comparative Analysis. Curr Tissue Eng. 2015;04:123-129.

[50] Takezawa T, Ozaki K and Takabayashi C. Reconstruction of a Hard Connective Tissue Utilizing a Pressed Silk Sheet and Type-I Collagen as the Scaffold for Fibroblasts. Tissue Eng. 2007;13:1357-66.

[51] Wu X, Black L, Santacana-Laffitte G and Patrick, CW. Preparation and assessment of glutaraldehydecrosslinked collagen-chitosan hydrogels for adipose tissue engineering. J Biomed Mater Res Part A. 2007;81A:59-65.

[52] Heino J. The collagen family members as cell adhesion proteins. BioEssays. 2007;29:1001-10.

[53] Jokinen J, Dadu E, Nykvist P, Käpylä J, White DJ, Ivaska J, et al. Integrin-mediated cell adhesion to type I collagen fibrils. J Biol Chem 2004;279:31956-63.

[54] Kalaskar DM and Alshomer F. Micro- and Nanotopographical Cues Guiding Biomaterial Host Response. In: Ed Lee, Yoo \& Atala, editors. In Situ Tissue Regeneration: Host Cell Recruitment and Biomaterial Design. Academic Press; 2016. P. 137-63.

[55] Mitra J, Tripathi G, Sharma A and Basu B. Scaffolds for bone tissue engineering: role of surface patterning on osteoblast response. RSC Adv. 2013;3:11073.

[56] Taylor P, Movasaghi Z, Rehman S and Rehman I. Fourier Transform Infrared ( FTIR ) Spectroscopy of Biological Tissues. Applied Spectroscopy Reviews. 2008;43:37-41.

[57] Nudelman F, Lausch AJ, Sommerdijk NAJM and Sone ED. In vitro models of collagen biomineralization. J Struct Biol. 2013;183:258-69.

[58] ISO 13779-3:2018. Available from: https://www.iso.org/standard/64618.html

\section{Figures}


(a)

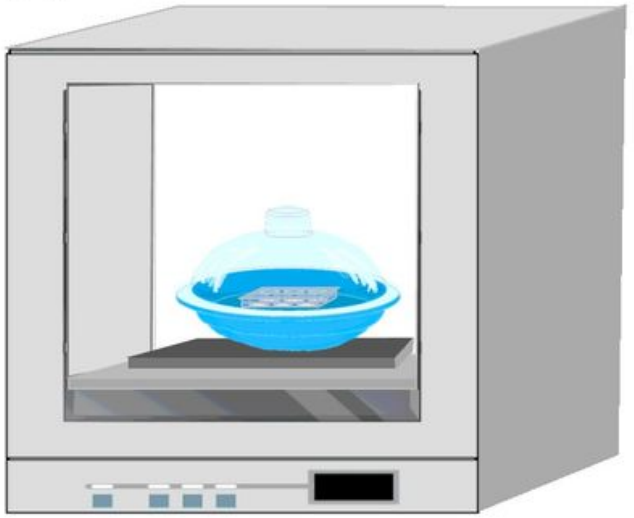

(b) $)_{50}$
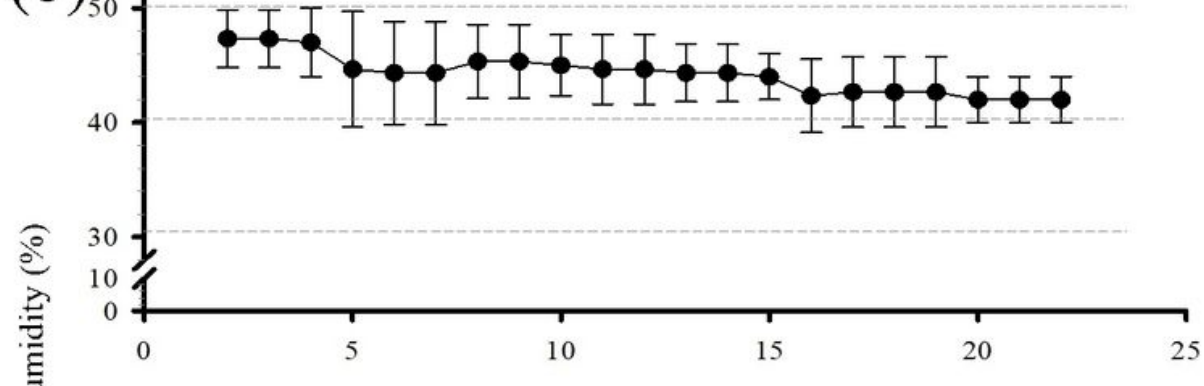

(c)

60
50
40
30
10
0

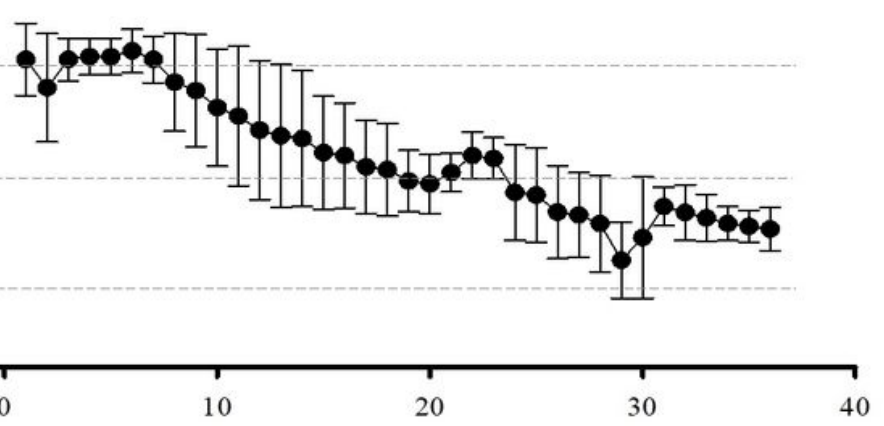

Days

\section{Figure 1}

Matryoshka system (a) assemblage. Daily behavior of the relative humidity percentage (b) at the stabilization phase and (c) in the presence of $6 \mathrm{~mL}$ of collagen gel.

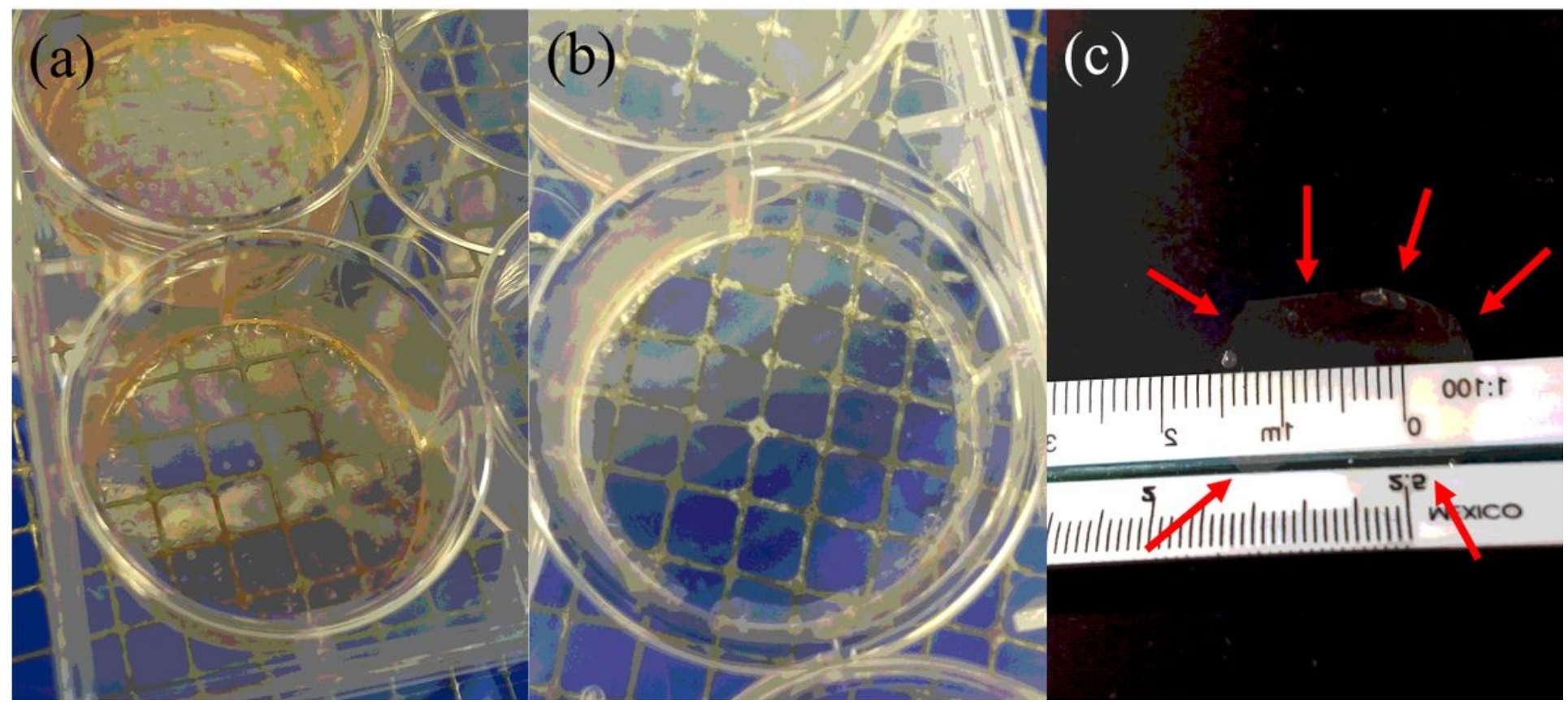

Figure 2 
Desiccation process: (a) gel (upper well) and membrane with phenol red; (b) rinsed membrane at day 37; and (c) membrane removed from the cast and dried at room temperature.

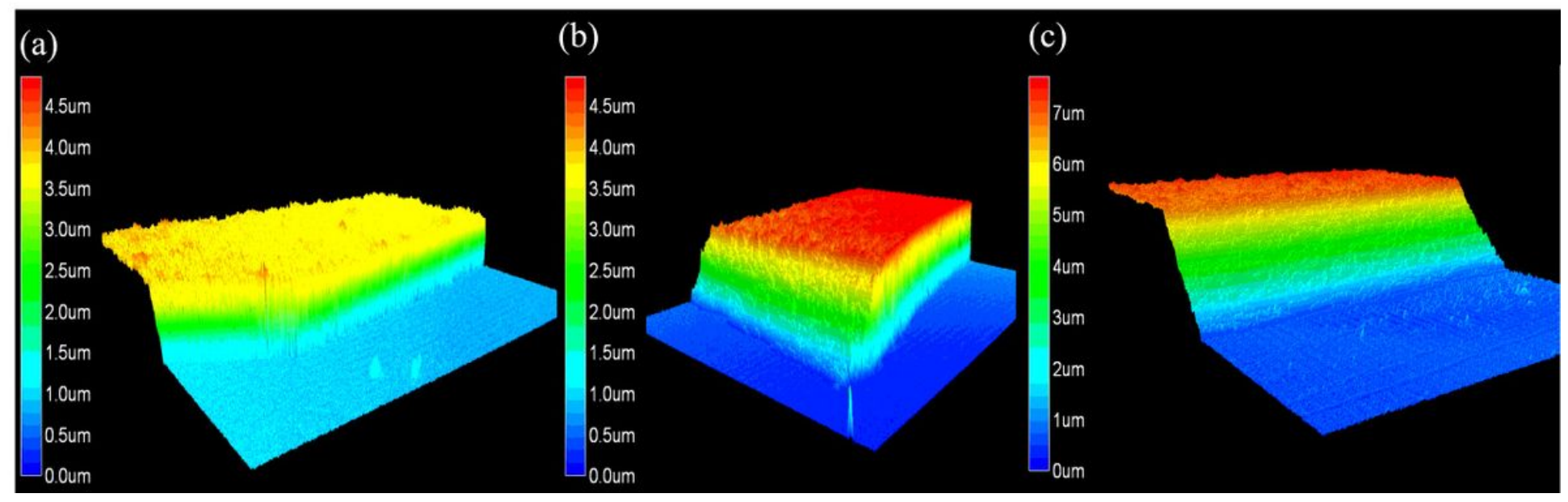

\section{Figure 3}

Representative surface and width evaluation using material confocal microscopy: (a) 1x, (b) 2x, and (c) $3 x$ collagen membranes $(n=3)$. 


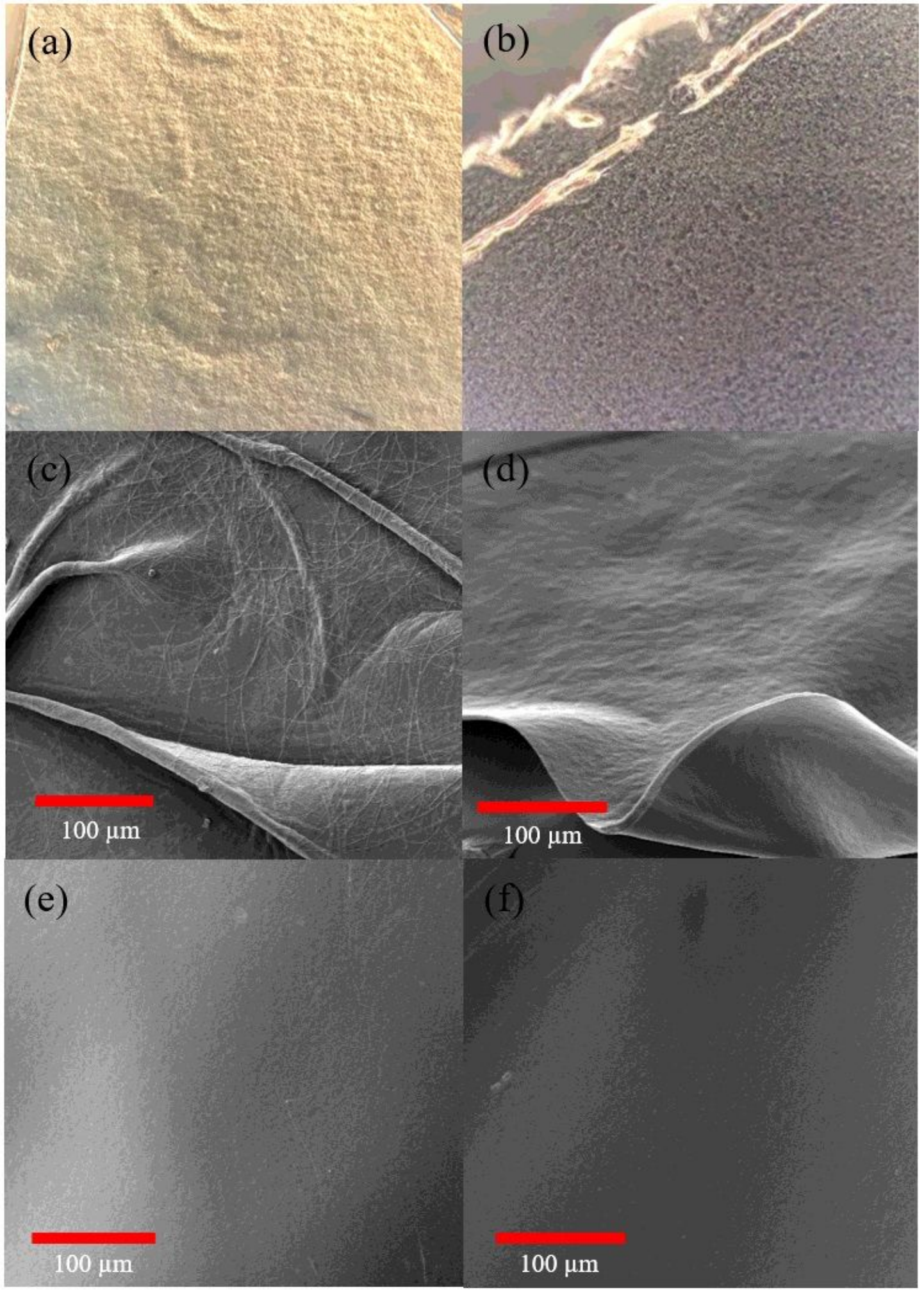

\section{Figure 4}

Contrast microscopy (10x image) of 37-day desiccated 1x membrane (a) with 20 min of hydration or (b) dry conditions. Surface micrograph of (c) a 7-day desiccated membrane and 37-day desiccated (d) 1x, (e) $2 x$, and (f) $3 x$ membranes. 


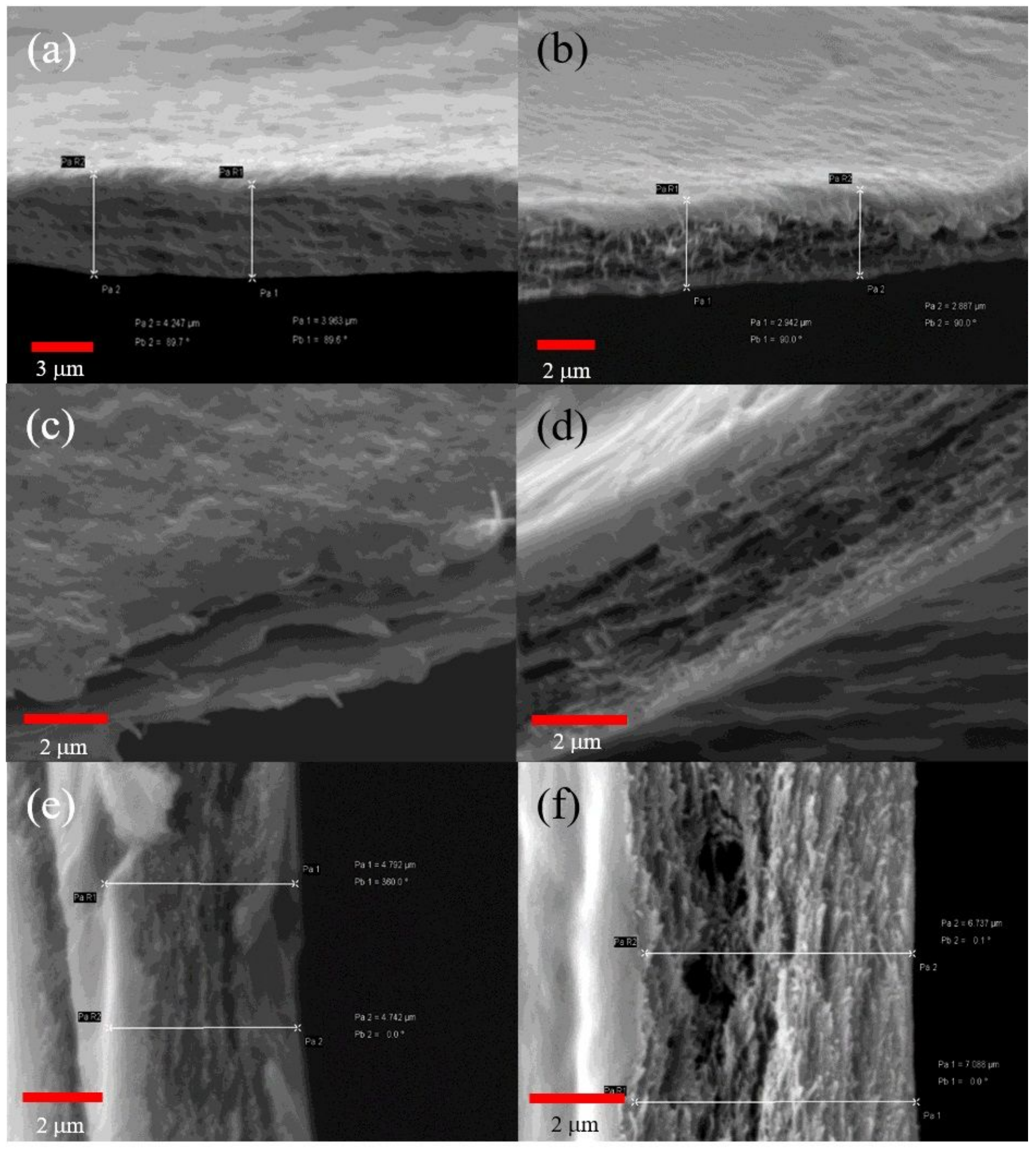

\section{Figure 5}

SEM transversal-view micrograph. Membrane at $1 x$ concentration (a) with 20 min of previous hydration and (b) in dry conditions. Detail of the laminar structure in (c) wet and (d) dry condition; (e) $2 x$ and (f) $3 x$ concentrations. 


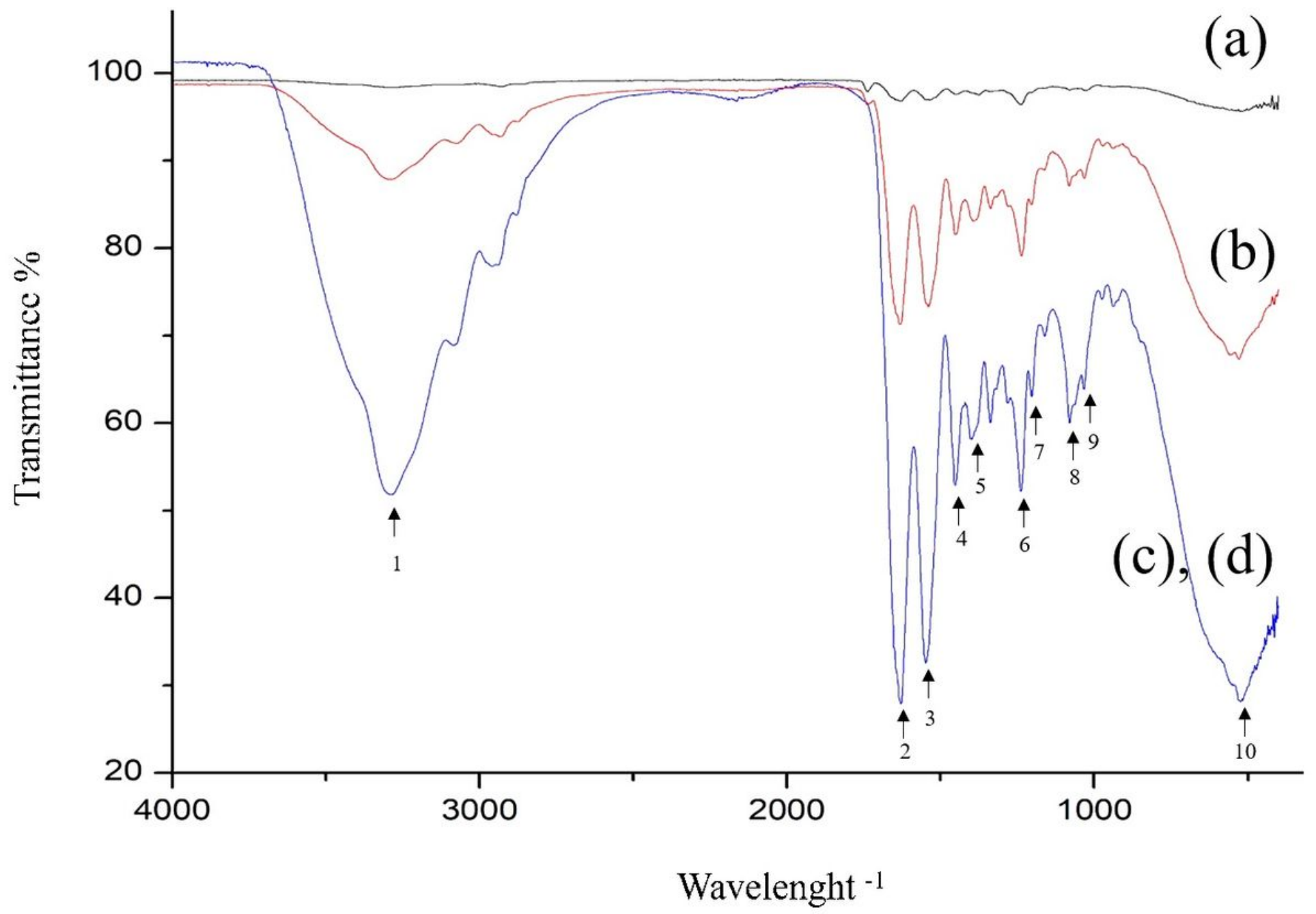

Figure 6

FTIR spectra of (a) pure collagen type 1, (b) 1x collagen membranes, (c) $2 x$ collagen membranes, and d) $3 x$ collagen membranes. 


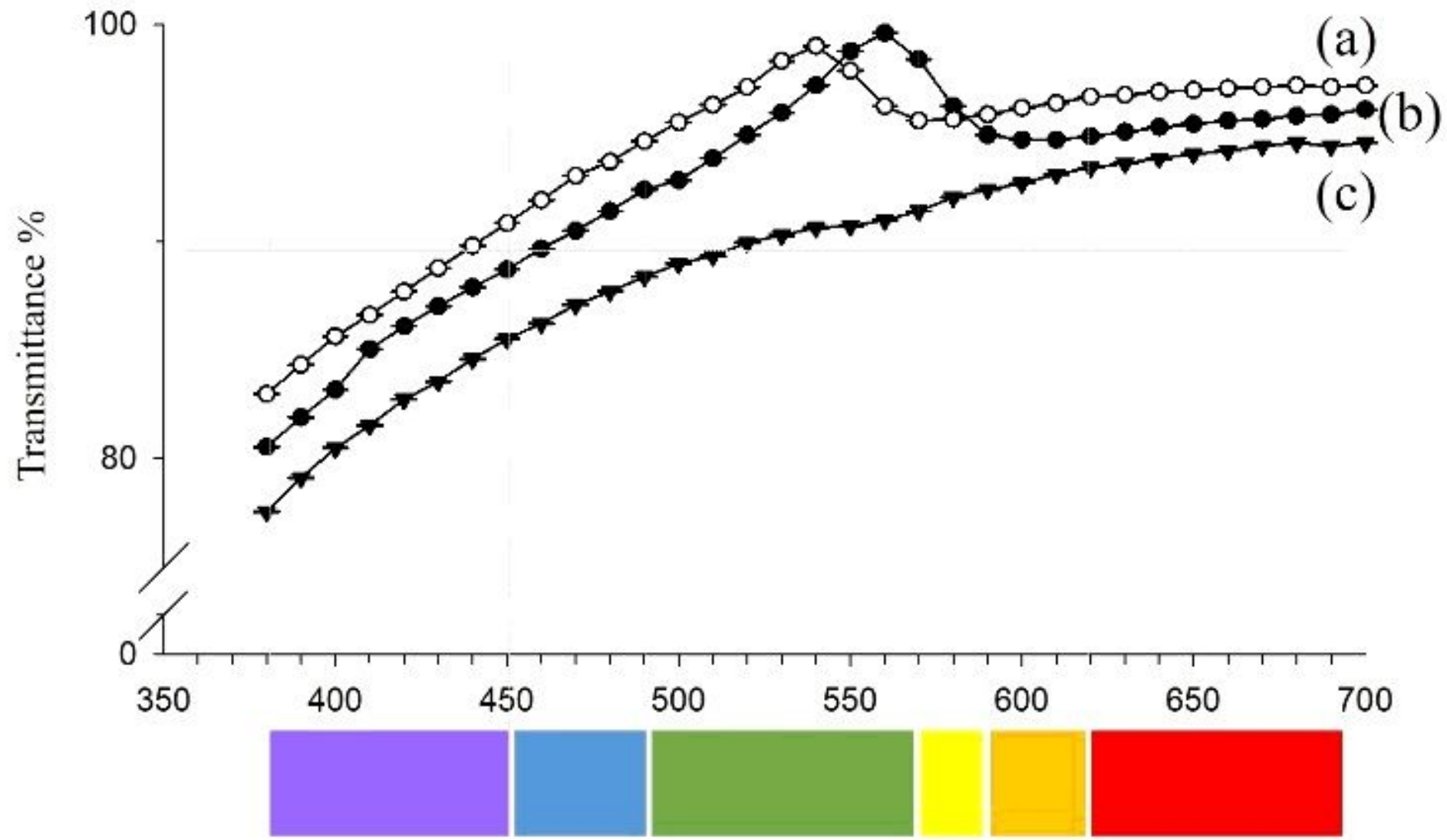

Wavelength

Figure 7

Percent transmittance of membranes fabricated using varying collagen volumes. (a) $2 x$ (b) $1 x$, and (c) $3 x$ membranes. 


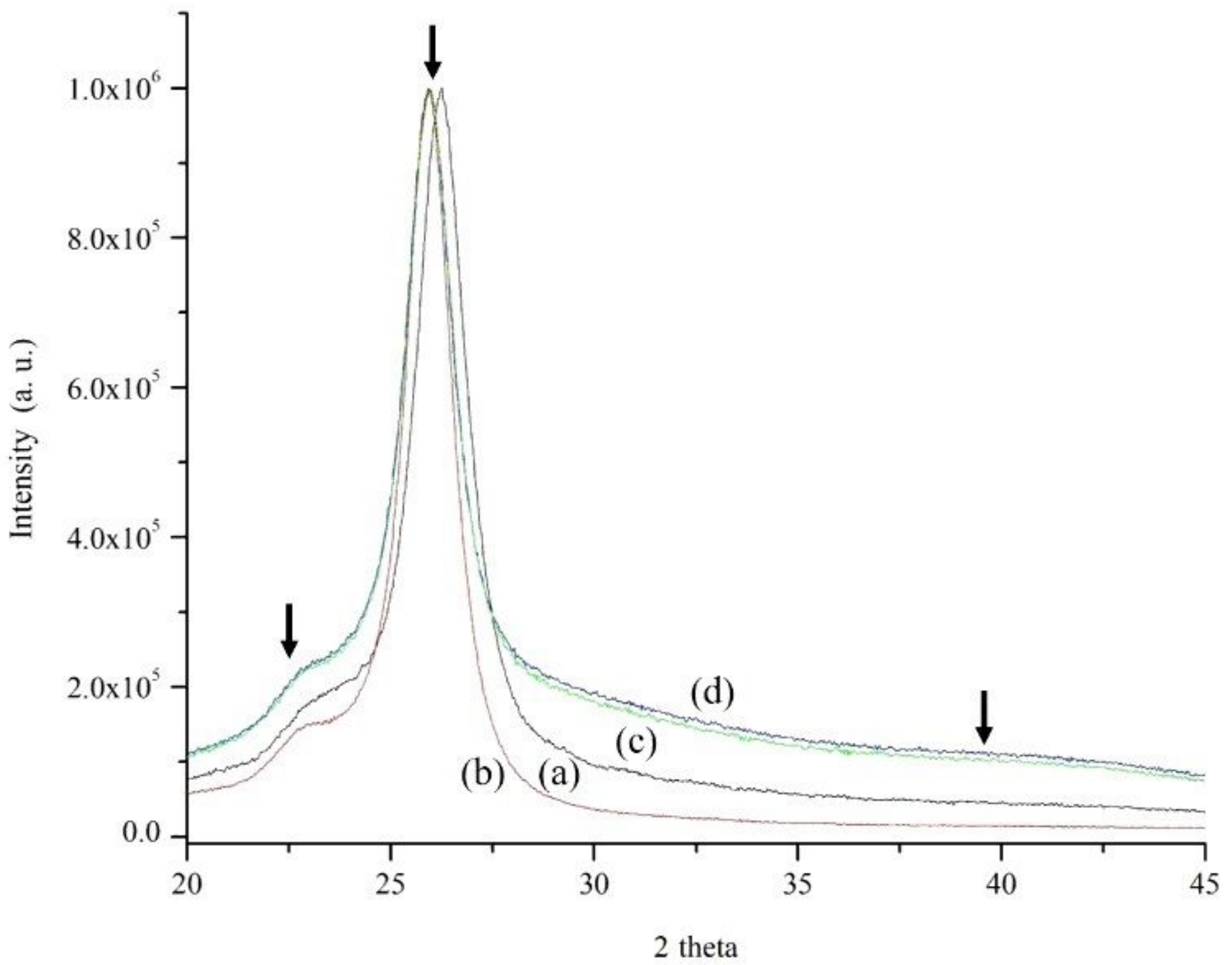

Figure 8

X-ray diffraction patterns of (a) collagen type 1 and (b) $1 x$, (c) 2x, and (d) $3 x$ collagen membranes. A $0.6^{\circ}$ offset was introduced to detect each pattern clearly. The arrows indicate the $22^{\circ}, 25.9^{\circ}, 39.8^{\circ}$ humps for amorphous collagen and the two hydroxyapatite planes, respectively. 